\title{
A influência da comunicação interna na inovação nas organizações: uma revisão sistemática integrativa
}

The influence of internal communication on innovation in organizations: an integrative systematic review

La influencia de la comunicación interna en la innovación en las organizaciones: una revisión sistemática integradora

\section{Kleiton Luiz Nascimento Reis}

- Doutorando em Engenharia e Gestão do Conhecimento pela Universidade Federal de Santa Catarina (UFSC).

- $\quad$ Mestre em Jornalismo pela UFSC.

- $\quad$ Especialista em Gestão da Comunicação em Mídias Digitais pela Faculdade Senac Florianópolis.

- $\quad$ Professor de Marketing na Faculdade Senac Florianópolis.

- E-mail: kleitonluiz.reis@gmail.com

\section{(9) Maria José Baldessar}

- Doutora em Ciências da Comunicação pela Escola de Comunicações e Artes da Universidade de São Paulo (ECA-USP).

- Professora do Programa de Pós-Graduação em Engenharia e Gestão do Conhecimento da USFC.

- Professora do Programa de Pós-Graduação em Estudos da Tradução da UFSC

- Professora do curso de graduação de Jornalismo da UFSC.

- E-mail: mbaldessar@gmail.com 


\section{Resumo}

A comunicação interna é o elemento fundamental na inovação nas organizações? Para responder a esta pergunta, este artigo buscou verificar na base de dados Scopus publicações sobre tais temas e descobrir qual é a influência da comunicação interna na inovação nas organizações. Para tanto, foi feita uma revisão sistemática integrativa em dez das 195 publicações encontradas. A análise revelou que, de fato, a comunicação interna influencia na inovação e é responsável por, por exemplo, difundir ideias e enriquecê-las.

\section{PALAVRAS-CHAVE: COMUNICAÇÃO INTERNA •INOVAÇÃO • REVISÃO SISTEMÁTICA INTEGRATIVA.}

\section{Abstract}

Is the internal communication a fundamental element in innovation in organizations? To answer this question, this paper aimed to check in the Scopus database for publications about those topics and to find out what is the impact of internal communication on innovation in organizations. For this, we conducted an integrative systematic review in ten of the 195 publications found. The analysis revealed that internal communication does impact innovation and is responsible for, for example, spreading ideas and enriching them.

\section{KEYWORDS: INTERNAL COMMUNICATION・INNOVATION・INTEGRATIVE SYSTEMATIC REVIEW.}

\section{Resumen}

¿Es la comunicación interna un elemento clave en la innovación organizacional? Para responder a esa pregunta, este artículo buscó en la base de datos Scopus publicaciones sobre comunicación interna e innovación para descubrir la influencia de la comunicación interna sobre la innovación organizacional. Para ello, se realizó una revisión sistemática integradora en diez de las 195 publicaciones encontradas. El análisis reveló que, de hecho, la comunicación interna influye en la innovación y es la responsable de difundir ideas y enriquecerlas. 
A inovação tem sido considerada essencial para que as empresas consigam sobreviver e se manter competitivas (Linke; Zerfass, 2011; Trott, 2012). Devido a sua importância para as organizações, os estudos sobre inovação empresarial cresceram substancialmente nas últimas décadas (Crossan; Apaydin, 2009). Estudos como o de Buckler (1997) e o de Linke e Zerfass (2011) consideram a inovação como um processo e, portanto, afirmam que ela não deve ser encarada apenas como a concepção de uma nova ideia ou uma ação única, mas como a integração de uma série de processos subjacentes que se inter-relacionam para que seja possível inovar (Myers; Marquis, 1969).

Nessa perspectiva, a comunicação interna aparece como um dos processos subjacentes ao processo de inovação (Damanpour, 1991) e deve ser coordenada entre vários grupos e incorporar novas atividades que visem lidar com os desafios propostos pelo processo de inovação nas organizações (Linke; Zerfass, 2011). Tal concepção coloca a comunicação interna como um fator crítico para a inovação (García-Morales; Matías-Reche; Verdú-Jover, 2011; Linke; Zerfass, 2011) e a condição fundamental para que as organizações possam crescer (Lemos, 2011). Por isso, a relação entre a comunicação interna e a inovação organizacional tem sido estudada por vários autores, que observam os aspectos positivos e negativos tanto na presença quanto na ausência de certas características e qualidades (García-Morales; Matías-Reche; Verdú-Jover, 2011).

Direcionada para os funcionários da organização (Lemos, 2011), a comunicação interna visa tornar viável a comunicação entre a empresa e seus empregados por meio da utilização de ferramentas de comunicação institucional e, às vezes, da comunicação com o mercado (Kunsch, 2003) para engajar os funcionários com as ações e estratégias da organização, se comunicar com eles, convencê-los de algo ou serem leais e aumentar a satisfação que sentem com o trabalho (Linke; Zerfass, 2011).

Na inovação, o objetivo da comunicação interna é aumentar a quantidade e facilitar o espalhamento de novas ideias na organização, assim como criar um ambiente favorável à sua sobrevivência (Damanpour, 1991) e auxiliar no processo de implantação (García-Morales; Matías-Reche; Verdú-Jover, 2011). Em decorrência de sua importância para a organização e para a eficácia dos processos de inovação, surge a necessidade de entender como a comunicação interna impacta os processos de inovação.

Com base nessa problemática, o objetivo deste artigo é verificar na base de dados Scopus publicações sobre comunicação interna e inovação e descobrir qual é a influência da primeira sobre a última. Para que fosse possível alcançar tal objetivo, recorreu-se à utilização do método de revisão sistemática integrativa da literatura, que visa mapear as pesquisas já realizadas sobre o tema para justificar as novas intervenções e a busca por mais evidências científicas sobre determinado assunto (Evans; Pearson, 2001).

Inicialmente, este trabalho foi estruturado em quatro seções. A primeira é esta introdução, seguida de uma apresentação sobre os procedimentos metodológicos e os resultados quantitativos da revisão sistemática da literatura. A terceira seção apresenta as análises dos estudos sobre os tópicos que são alvo desta investigação. Por fim, a última seção trata das considerações finais deste estudo. 


\section{INOVAÇÃO NAS ORGANIZAÇÕES}

A visão de que a inovação traz vantagem competitiva para as organizações não é recente. Trott (2012) lembra que desde 0 início do século XX já se estudava o potencial das inovações de garantir a sobrevivência das organizações, mas que estudos mais recentes têm aprimorado a compreensão sobre a gestão da inovação nas empresas. Para o autor, a inovação trata-se da aquisição e da aplicação de conhecimentos que proporcionem o desenvolvimento de novos produtos que satisfaçam as necessidades dos consumidores.

A partir dessa compreensão da inovação como a aplicação de conhecimentos adquiridos, Trott (2012) defende que "a inovação precisa ser vista no contexto das organizações e como processo dentro delas" (Trott, 2012, p.10), já que a aquisição de conhecimento nas empresas é constante e a inovação ocorre a partir da integração de vários outros processos organizacionais. É por isso que o autor aponta que existem uma série de atividades organizacionais que influenciam o sucesso das inovações.

Coral et al. (2008) afirmam que, para que se tenha controle da trajetória de implementação das inovações, deve haver planejamento e o processo de desenvolvimento deve ser sistematizado. Ainda, para os autores, o planejamento da inovação deve partir do planejamento estratégico da organização, considerando aspectos tanto da tecnologia quanto do mercado. Nesse sentido, a proposta de inovação deve ser coerente com os objetivos de negócio da empresa e "estabelecer metas claras quanto ao desenvolvimento de novos produtos, implementação de projetos e investimentos em pesquisa e desenvolvimento, aquisição de tecnologia e capacitação para inovação" (Coral et al., 2008, p.32).

Para Coral et al. (2008), uma comunicação interna eficiente ainda é um dos principais desafios a serem enfrentados em um processo de gestão da inovação. Da mesma forma, o relacionamento entre os indivíduos e as equipes envolvidas no desenvolvimento das inovações é crucial para que os projetos sejam discutidos, priorizados e implementados. Assim, se 0 processo de inovação acontece, em última instância, devido à atuação de vários indivíduos, e se as atividades organizacionais influenciam o potencial inovador da empresa, é necessário garantir que estes indivíduos estejam alinhados e motivados para implantar uma inovação, tendo a comunicação interna da empresa como ferramenta.

\section{COMUNICAÇÃO INTERNA}

Devido ao potencial de impacto na organização, Kunsch (2003) afirma que a comunicação organizacional se tornou atividade estratégica e, por isso, precisa ser planejada e estar alinhada aos objetivos da organização (Corrêa, 2008). Para Kunsch (2003), a comunicação organizacional trata-se da integração da comunicação institucional, da comunicação com o mercado, da comunicação administrativa e da comunicação interna. Neste conjunto, a comunicação interna é aquela direcionada para os funcionários da organização e que visa tornar viável a troca de informações entre a empresa e seus empregados, a fim de engajá-los com as ações e estratégias da organização e aumentar sua satisfação com o trabalho (Linke; Zerfass, 2011).

É por meio da comunicação interna que os funcionários conhecem os objetivos estratégicos da organização, bem como a sua missão, visão, valores, propósitos e a qualidade de seus produtos e serviços (Clemen, 2005; Drucker, 1964). Ademais, a comunicação interna colabora para a adaptação, compreensão e engajamento dos funcionários nas ideias e ações estratégicas da empresa (Macarenco; Terciotti, 2013). Somam-se a tal tipo de comunicação as funções de melhorar o clima organizacional e de tornar os colaboradores mais produtivos, valorizados e respeitados (Cenerini, 2009). 
Em vista disso, coloca Brum (2010), a comunicação interna também é primordial nos momentos em que a empresa passa por mudanças e os funcionários se deparam com novos desafios, como quando estão desenvolvendo um novo produto ou processo. Neste momento, a comunicação assume novamente um papel estratégico para a adaptação e o engajamento dos funcionários diante ados novos desafios.

\section{PROCEDIMENTOS METODOLÓGICOS}

Para resolver o problema levantado por este artigo, foi preciso realizar um estudo descritivo a partir de um levantamento bibliográfico, realizado por meio da utilização do estudo bibliométrico e do processo de revisão sistemática integrativa da literatura. De acordo com Araújo (2006), a bibliometria tem como objetivo medir a produção e a disseminação de conhecimento científico por meio de técnicas quantitativas e estatísticas. Já a revisão integrativa, segundo Botelho, Cunha e Macedo (2011, p.127), "possibilita a síntese de vários estudos já publicados, permitindo a geração de novos conhecimentos, pautados nos resultados apresentados pelas pesquisas anteriores".

Em outras palavras, enquanto o estudo bibliométrico auxilia na medição dos índices de produção científica, a revisão integrativa permite analisar o que já foi estudado sobre um tema e extrair as conclusões necessárias para responder a uma pergunta.

Para realizar uma revisão integrativa da literatura, Lasserson, Thomas e Higgins (2019) afirmam que é necessário utilizar métodos explícitos e sistemáticos na seleção dos trabalhos a serem analisados, com o intuito de minimizar vieses do pesquisador na hora de realizar a revisão da literatura existente sobre o assunto.

Com base em diversos estudos sobre o tema, Botelho, Cunha e Macedo (2011) destacam quais etapas é preciso seguir para empreender uma revisão relevante. Segundo os autores, na primeira etapa da revisão sistemática integrativa da literatura deve se elaborar um protocolo em que se possa identificar o tema e selecionar a questão proposta pela pesquisa. Define-se, também, a estratégia de busca, isto é, as regras para encontrar as informações que respondem à pergunta de pesquisa e a base de dados a ser consultada.

Neste trabalho, o tema a ser investigado é a comunicação interna nos processos de inovação nas organizações e a questão central é: Como a comunicação tem aparecido nos estudos sobre inovação nas organizações? Decidiu-se realizar a pesquisa utilizando a base de dados Scopus, pela sua abrangência no que se refere a fontes de publicações técnicas e científicas e à disponibilidade de acesso gratuito à maioria dos artigos. Foram definidos como descritores as palavras-chave internal communication, organizational communication e innovation. A busca foi feita por meio dstring TITLE-ABS-KEY - internal communication ou organizational communication - e TITLE-ABS-KEY - innovation.

As regras da estratégia de busca determinaram que seriam mapeados os artigos em que aparecessem no título, no resumo ou nas palavras-chave os termos comunicação interna ou comunicação organização - incluindo suas variações em inglês -, juntamente com o termo inovação e sua variação em inglês. Nesta primeira busca, foram identificadas 196 publicações que utilizaram os descritores pré-selecionados no título, no resumo ou nas palavras-chave, e quatro eram publicações duplicadas.

Com uma breve análise bibliométrica dos resultados obtidos nesse primeiro levantamento, foi possível perceber que a quantidade de publicações sobre a influência da comunicação interna na inovação nas organizações cresceu expressivamente, apesar de algumas variações em alguns anos das últimas décadas (Gráfico 1). 


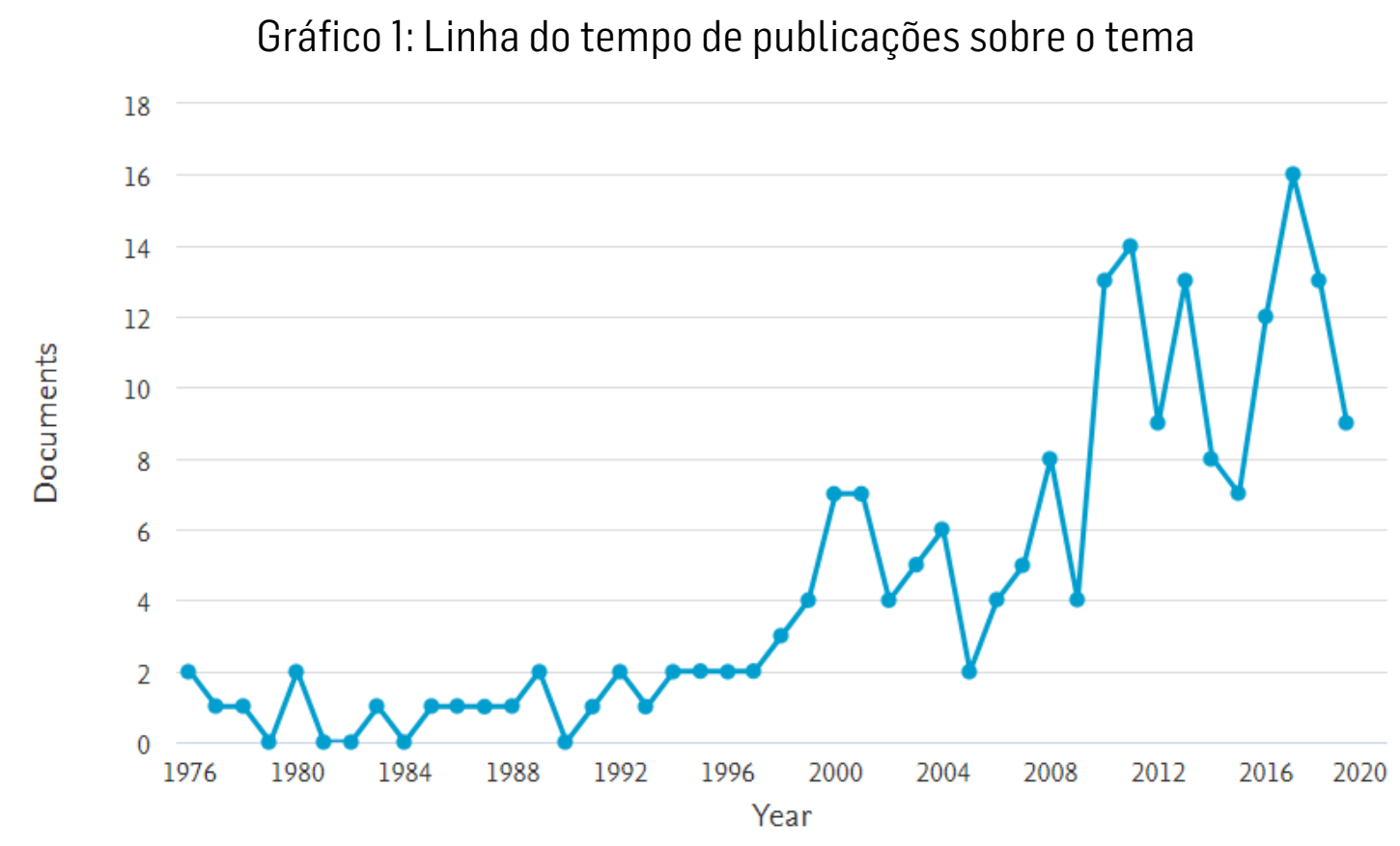

Fonte: Scopus.

O crescimento no número de publicações mostra ou que desde o início do século XXI houve um aumento no interesse sobre 0 tema, ou que, pelo menos, os estudos passaram a se atentar mais para a influência que a comunicação interna pode exercer durante as inovações que ocorrem nas organizações. Outro dado interessante desse levantamento prévio mostra que a Universidade do Sul da Califórnia é a instituição com o maior número de publicações sobre o tema (Gráfico 2).

Gráfico 2: Distribuição de publicações por universidades

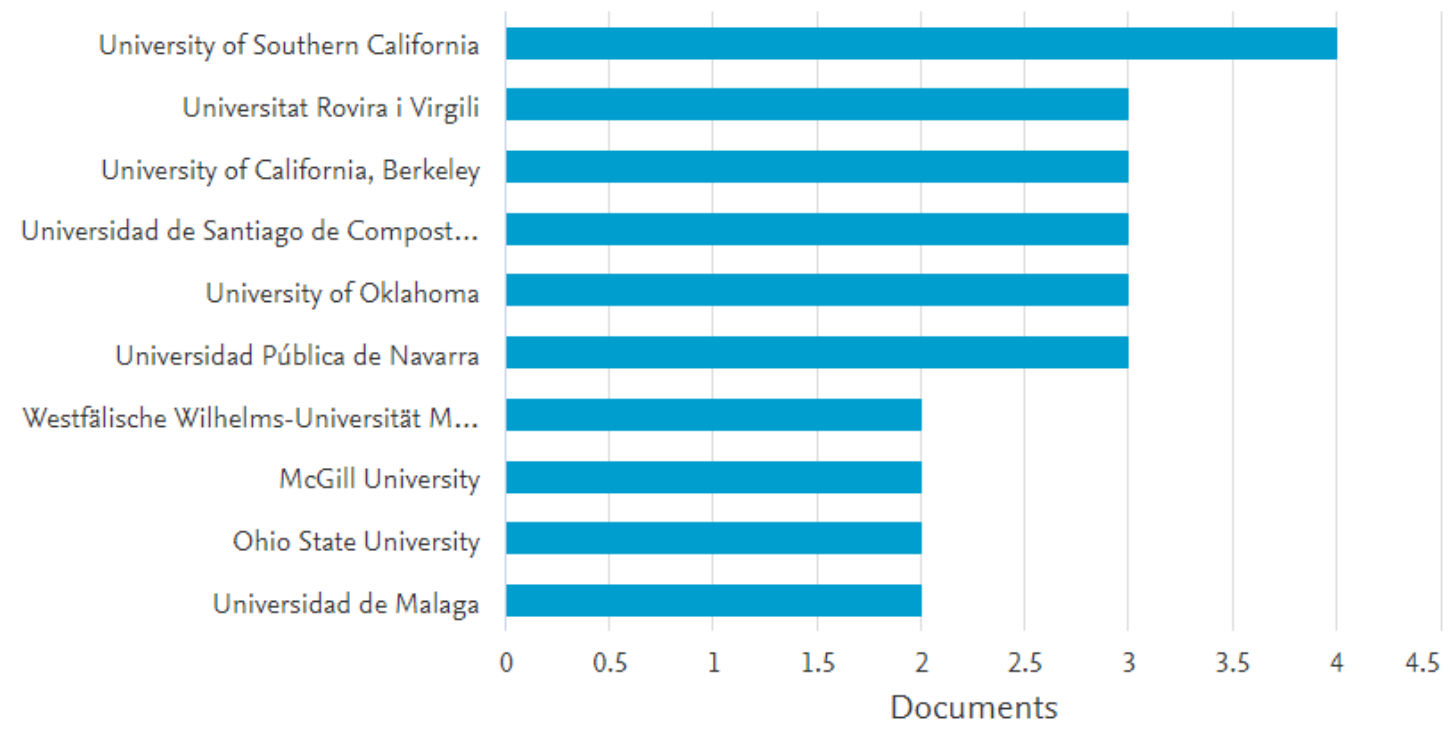

Fonte: Scopus.

Além disso, há uma relevante variedade de áreas cujos estudos trataram sobre o tema. É possível perceber, como mostra 0 Gráfico 3, que as áreas de administração de empresas, ciências sociais e ciências da computação concentram mais de $50 \%$ das publicações. 
Gráfico 3: Distribuição de publicações por área do conhecimento

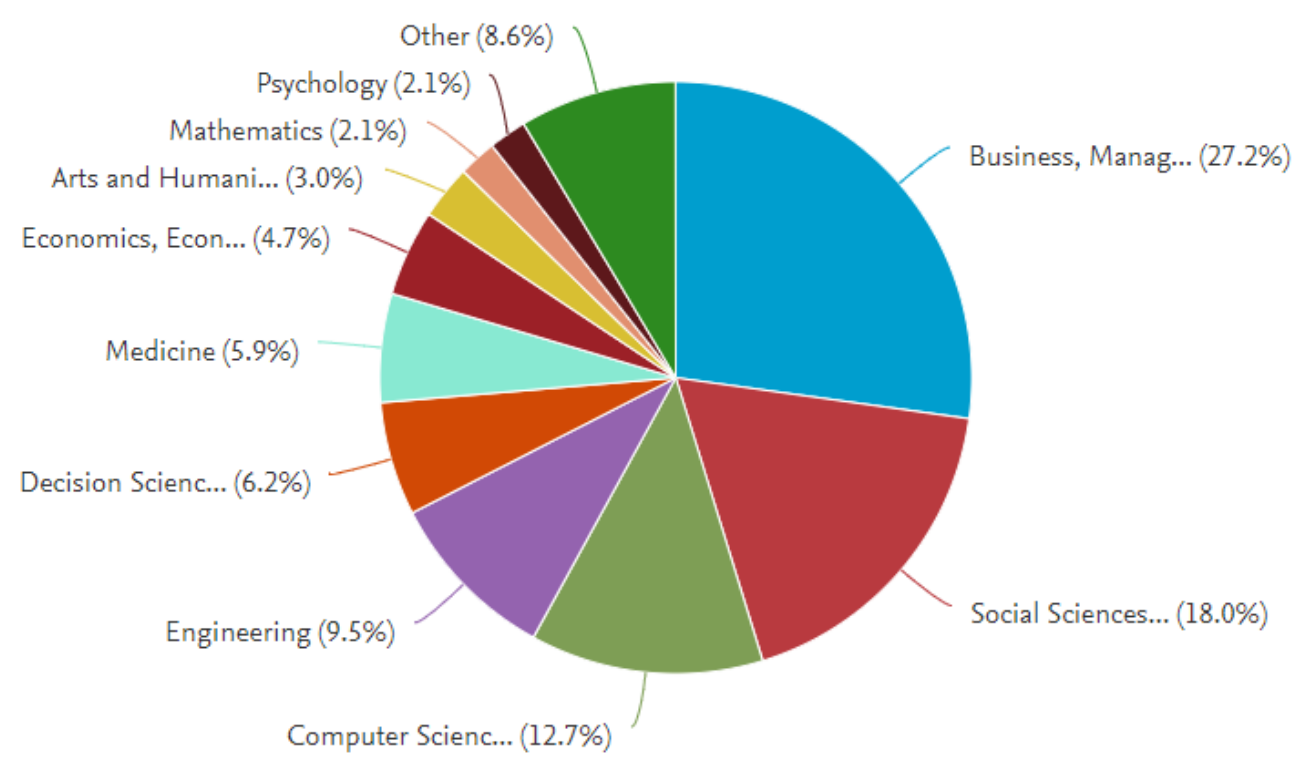

Fonte: Scopus.

Por último, percebe-se que o tema é pesquisado, principalmente, por instituições americanas e européias, embora também haja contribuições da Austrália, da China e do Canadá (Gráfico 4).

Gráfico 4: Distribuição das publicações por país

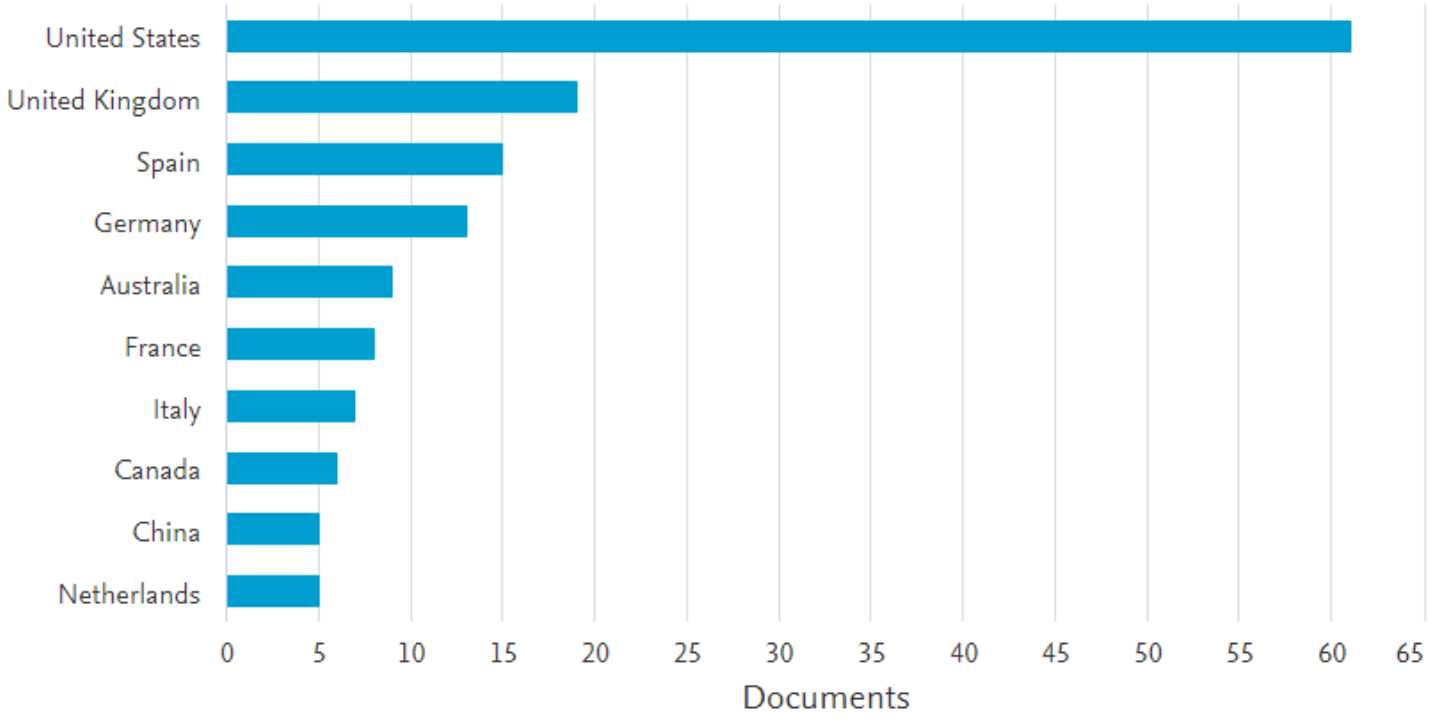

Fonte: Scopus.

Na segunda etapa da revisão sistemática integrativa, segundo Botelho, Cunha e Macedo (2011), o pesquisador deve estabelecer os critérios de inclusão e exclusão para, então, proceder com as análises dos artigos. Neste estudo, utilizou-se como critério de inclusão estudos cujo foco foi analisar a comunicação interna nos processos de inovação e, como critérios de exclusão, a impossibilidade de acessar o artigo completo e publicações que não estavam em língua inglesa, espanhola ou portuguesa.

A terceira etapa, de acordo com os autores, trata-se da identificação dos estudos pré-selecionados e selecionados. Neste momento, o pesquisador avalia os títulos, os resumos e as palavras-chave para selecionar os artigos que farão parte da revisão.

Como já dito, foram selecionados para este trabalho os artigos que apresentavam no título, no resumo e nas palavras-chave os termos comunicação interna ou comunicação organizacional ou suas variações em inglês. Também foi utilizado como critério de seleção o fato de os artigos apresentarem ou não como tema principal a discussão da relação da comunicação 
interna com o processo de inovação. Os artigos que apenas citavam a comunicação interna como atividade participante do processo e, consequentemente, não aprofundavam o tema, foram excluídos da análise. Esse requisito é importante para que seja possível responder à questão de pesquisa adotada.

Das publicações encontradas, a mais citada em outros trabalhos foi o artigo "External linkages and innovation in small and medium-sized enterprises", de Rothwell e Dodgson (1991), mas este trabalho não estava disponível para download para que pudesse ser analisado. Assim, nesta etapa de identificação, os artigos foram analisados e, considerando os critérios de exclusão, dez artigos foram selecionados para a análise (Quadro 1).

Quadro 1: Artigos selecionados para análise

\begin{tabular}{|c|c|c|}
\hline Autor & Título do artigo & Ano \\
\hline $\begin{array}{l}\text { Suh, Jiwon; Harrington, } \\
\text { James; Goodman, Doug. }\end{array}$ & $\begin{array}{l}\text { Understanding the link between organizational } \\
\text { communication and innovation: an examination of public, } \\
\text { nonprofit, and for-profit organizations in South Korea. }\end{array}$ & 2018 \\
\hline Glińska-Neweś, Aldona et al. & $\begin{array}{l}\text { Innovations among people: how positive relationships } \\
\text { at work can trigger innovation creation. }\end{array}$ & 2017 \\
\hline Linke, Anne; Zerfass, Ansgar. & $\begin{array}{l}\text { Internal communication and innovation culture: } \\
\text { developing a change framework. }\end{array}$ & 2011 \\
\hline $\begin{array}{l}\text { García-Morales, Víctor; } \\
\text { Matías-Reche, Fernando; } \\
\text { Verdú-Jover, Antonio. }\end{array}$ & $\begin{array}{l}\text { Influence of internal communication on technological } \\
\text { proactivity, organizational learning, and organizational } \\
\text { innovation in the pharmaceutical sector. }\end{array}$ & 2011 \\
\hline Zhang, Gang; Gao, Ruoyang. & $\begin{array}{l}\text { Modularity and incremental innovation: the roles of } \\
\text { design rules and organizational communication. }\end{array}$ & 2010 \\
\hline $\begin{array}{l}\text { François, Jean-Paul; Favre, } \\
\text { Florent; Negassi, Syoum. }\end{array}$ & Competence and organization: two drivers of innovation. & 2002 \\
\hline Ulijn, Jan et al. & $\begin{array}{l}\text { Innovation, corporate strategy, and cultural context: what is } \\
\text { the mission for international business communication? }\end{array}$ & 2000 \\
\hline Fairtlough, Gerard. & Organizing for innovation: compartments, competences and networks. & 1994 \\
\hline Fuchigami, Katsuyoshi & $\begin{array}{c}\text { A study of organizational communication in a school } \\
\text { during the pre- and post-innovation periods. }\end{array}$ & 1993 \\
\hline Digman, Lester A. & Organizational factors related to technology transfer and innovation. & 1977 \\
\hline
\end{tabular}

Fonte: Elaborado pelos autores.

As próximas etapas da revisão integrativa, a saber, a categorização dos estudos selecionados, cujo intuito é "sumarizar e documentar as informações extraídas dos artigos científicos encontrados nas fases anteriores" (Botelho; Cunha; Macedo, 2011, p.131), e a análise e interpretação dos resultados, ou seja, a interpretação dos dados para identificar os gaps de conhecimento e sugerir novas abordagens, serão melhor explicadas nas seções seguintes. 


\section{ANÁLISE INTEGRATIVA: A COMUNICAÇÃO INTERNA NA INOVAÇÃO}

A partir da leitura das publicações selecionadas, pôde-se perceber que a comunicação interna tem influência direta na maneira como as empresas gerenciam a inovação. Contudo, observou-se também que pouca atenção tem sido dada para este tema, visto a pouca quantidade e o tempo de publicação dos trabalhos.

Apesar de os trabalhos analisados concordarem que pouco esforço de pesquisa tem sido direcionado para compreender a influência da comunicação na inovação (García-Morales; Matías-Reche; Verdú-Jover, 2011; Linke; Zerfass, 2011), Linke e Zerfas (2011) afirmam que está crescendo a importância da área de comunicação para a inovação. Isso porque a inovação, para que aconteça, demanda comunicação entre várias pessoas da empresa, em todos os níveis. Logo, a comunicação interna e a comunicação interpessoal se tornam imprescindíveis para inovar (García-Morales; Matías-Reche; Verdú-Jover, 2011), para facilitar a mudança contínua (Suh; Harrington; Goodman, 2018) e para criar uma cultura de inovação na empresa (Linke; Zerfass, 2011).

Desde a década de 1980, Gerard Fairtlough (1994) já previa que a comunicação teria um papel fundamental na organização da inovação nas empresas. Para o autor, a comunicação é uma das responsáveis pelo desenvolvimento das redes dentro das organizações, capazes de criar um ambiente propício à inovação. Antes disso, Digman (1977) já relembrava estudos anteriores que buscavam compreender quais eram as características de empresas consideradas inovadoras. 0 levantamento do autor considerou que existem fatores que aumentam a utilização e a geração de inovações e a qualidade da organização, sendo a comunicação interna efetiva um deles. Esse aspecto é ressaltado ainda por outros dois estudos que 0 autor destaca. 0 primeiro diz respeito a uma pesquisa feita por Stewart, em 1967, sobre um frameworkconceitual de parâmetros para indicar as características de um negócio de base tecnológica, em que aponta ser necessário um alto grau de integração entre as áreas de pesquisa e desenvolvimento, fabricação, marketing e cliente, e que essa integração depende da comunicação e cooperação de várias funções.

O segundo estudo destacado por Digman (1977) é o relatório da Academia Nacional de Ciências para a Câmara dos Deputados dos Estados Unidos da América (EUA), no qual o autor mostra que "existe comunicação completa em todas as etapas da pesquisa e processo de desenvolvimento, desde a pesquisa original até a aplicação final" (Digman, 1977, p.67, tradução nossa).

François, Favre e Negassi (2002) reforçam a investigação de Digman (1977). Para os autores, o sucesso da inovação nas organizações depende diretamente de uma série de fatores organizacionais, entre eles a comunicação interna. Além disso, buscam comprovar em sua publicação que tais fatores são responsáveis pelo sucesso da inovação também em pequenas e médias empresas. Segundo os autores, havia ainda uma concepção corrente, baseada na proposta de Schumpeter, de que somente grandes empresas poderiam inovar: "Quando a estrutura organizacional é integrada ao modelo de Schumpeter com flexibilidade, reatividade, tomada de riscos, comunicação interna e o estabelecimento de uma estratégia, a importância do tamanho da empresa diminui pela metade" (François; Favre; Negassi, 2002, p.262).

De forma abrangente, um estudo conduzido por Ulijn et al. (2000) a partir de uma revisão da literatura sobre a comunicação interna e sua influência na inovação nas organizações mostrou que muitas pesquisas anteriores já haviam se debruçado sobre o tema, trazendo vários dos aspectos trabalhados por outros autores nos anos seguintes. 0 trabalho dos autores aponta 0 uso da comunicação interna, por exemplo, para a difusão de ideais na organização, para a adoção de mudanças dentro da organização, a implementação de estratégias e de inovações na organização e a criação de redes de comunicação interna, visando, assim, criar ambientes de discussão sobre inovações e ideias inovadoras. A análise da literatura disponível na época levou os autores a concluírem que a comunicação interna é influenciada pela cultura nacional, corporativa e profissional e que, por sua vez, impacta as estratégias de inovação das empresas. A esse respeito, o autor propõe que a comunicação deve 
ser trabalhada de formas diferentes em cada cultura e levar em consideração suas variações para, assim, permitir que a inovação aconteça de forma efetiva na organização.

Sobre como a comunicação deve ser trabalhada nas organizações, tanto García-Morales, Matías-Rech e Verdú-Jover (2011) quanto Linke e Zerfass (2011) defendem em seus estudos que a comunicação deve acontecer por uma via de mão dupla, pois dessa forma poderá proporcionar o sucesso da comunicação interna e aumentar o engajamento e a satisfação dos funcionários da organização com seus trabalhos individuais e com a empresa como um todo. Para os autores, essa concepção de bidirecionalidade da comunicação ajuda as empresas a construírem as interações sociais necessárias para uma melhor performance do processo de inovação nas organizações.

Nesse sentido, uma comunicação aberta e flexível, que busca considerar e entender a opinião de todos e combinar e integrar ideias, é essencial (García-Morales; Matías-Reche; Verdú-Jover, 2011). Mas apenas isso não é suficiente. A principal proposta do trabalho de Linke e Zerfass (2011), por exemplo, é que a comunicação interna deve deixar para trás o modelo generalista para dar lugar a um framework específico, que se adapte às condições - tipo de mensagem, tom, ferramentas etc. - de cada fase do processo de inovação - consciência, compreensão, aceitação e ação -, desempenhando, assim, não apenas a função de transmissão de informações, mas diversas outras, como a de agilizar processos de feedbacks cíclicos.

De forma mais prática, Zhang e Gao (2010) conduziram experimentos que mostram o impacto da comunicação no processo de inovação incremental. Os autores tratam a inovação como um processo constituído por módulos interdependentes, que devem ter regras para funcionar. No entanto, quando essas regras falham, entra em cena a comunicação, principalmente entre os gerentes dos módulos. Em seus experimentos, Zhang e Gao (2010) descobriram que, quando a comunicação não é eficaz, os envolvidos nos módulos do processo de inovação podem não saber o que está acontecendo e, assim, gerar distúrbios no processo. Por outro lado, os autores confirmam que, quando a comunicação é efetiva, além de melhorar a performance do processo, ainda promove um aprendizado para a equipe, principalmente no longo prazo. 0 estudo de Linke e Zerfass (2011), no entanto, identificou que as fases do processo de inovação podem acontecer ao mesmo tempo, por isso a comunicação deve atender a diferentes requisitos simultaneamente. Neste caso, García-Morales, Matías-Rech e Verdú-Jover (2011) alertam que a empresa deve ter o cuidado de não tornar a comunicação excessiva, pois isto possibilitaria que informações redundantes circulassem pela empresa.

Outra contribuição importante da pesquisa de García-Morales, Matías-Rech e Verdú-Jover (2011) é que a comunicação interna também ajuda a avaliar, assimilar, aplicar e expandir novos conhecimentos para melhorar a competitividade das empresas. Neste aspecto, os autores apontam que a tecnologia deve ser aliada da comunicação interna, pois ela pode ajudar a transformar o conhecimento tácito em explícito, o que é fundamental para o aprendizado organizacional e para a inovação. Os autores ressaltam que, com novos conhecimentos circulando pela organização, novas ideias podem surgir, tornando a comunicação interna responsável por espalhar, diversificar e fertilizar as novas ideias dentro da organização, bem como por promover a aceitação do resultado do processo de inovação e garantir o envolvimento dos integrantes da empresa em todas as fases do processo.

A comunicação interna não é responsável apenas pela difusão de ideias na organização; também exerce outro papel fundamental, ajudando a superar a resistência à inovação nas organizações, criando um clima organizacional propício, reduzindo as incertezas relacionadas a ela e desenvolvendo uma consciência mais ampla de que suas implicações serão mais benéficas do que manter as práticas organizacionais atuais (García-Morales; Matías-Reche; Verdú-Jover, 2011; GlińskaNeweś et al. , 2017). 
Devido a sua importância nos processos de inovação e seus impactos, que podem ser positivos ou negativos, García-Morales, Matías-Rech e Verdú-Jover (2011) defendem que a comunicação interna deve ser bem gerenciada e testada empiricamente. Em seus estudos empíricos em empresas farmacêuticas europeias e norte-americanas, quando testaram algumas hipóteses - entre elas a influência da comunicação interna e o processo de inovação -, os autores encontraram uma relação direta e significativa entre os dois elementos. Como resultado, os autores indicam que "métodos e canais eficazes de IC devem ser estabelecidos e usados por todos os envolvidos na inovação" (García-Morales; Matías-Reche; Verdú-Jover, 2011, p.170).

Nesta mesma linha, a investigação conduzida por Suh, Harrington e Goodman (2018) levou os autores a defenderem que a comunicação interna incentiva a inovação contínua por parte dos funcionários da empresa, gerando uma contribuição importante. Durante seus estudos em organizações sul-coreanas, eles descobriram que a comunicação interna demonstra ter influência direta na inovação orientada a funcionários do setor com fins lucrativos, mas que os impactos são mistos no setor sem fins lucrativos e que não há efeito no setor público. Outras contribuições importantes do estudo de Suh, Harrington e Goodman (2018) são: a quantidade de canais de comunicação utilizados na organização tem pouca influência na eficiência do processo de inovação; apenas dois tipos de reuniões entre os nove testados - as reuniões com o diretor executivo e 0 e-mail - têm marginalmente algum efeito na inovação orientada por funcionários; é importante ter um fluxo de comunicação ascendente, para que a alta gestão possa ouvir as demandas e ideias dos funcionários.

Com foco também no papel do funcionário na inovação, Glińska-Neweś et al. (2017) demonstraram que as relações positivas no trabalho são essenciais para a boa condução da inovação nas organizações. Nesse sentido, para os autores, a comunicação interna tem papel fundamental na inovação, pois influencia as relações interpessoais e sociais, incentivam a troca de informações frequentes e são responsáveis por disseminar o conhecimento pela organização.

\section{CONSIDERAÇÕES FINAIS}

Por meio da realização de uma revisão sistemática da literatura, com suporte da análise descritiva das publicações selecionadas a partir do protocolo estabelecido, foi possível perceber que os estudos sobre a influência da comunicação interna nos processos de inovação ainda são escassos, embora alguns autores tenham, de alguma forma, se aprofundado no tema. No entanto, análises preliminares das publicações encontradas antes que os critérios de exclusão fossem incluídos mostraram que alguns estudos sobre inovação acabaram percebendo que a comunicação impacta ou é impactada no processo, embora não tenham mergulhado no tema, visto que seus objetivos eram outros.

Ainda, foi possível notar que os estudos sobre a influência da comunicação interna na inovação foram conduzidos sob diferentes metodologias e procedimentos, como estudos teóricos, entrevistas, observações e levantamentos via questionários. É interessante notar, porém, que grande parte dos trabalhos foram produzidos a partir de pesquisas empíricas, trazendo, dessa forma, dados da realidade e mostrando como, de fato, a comunicação tem colaborado e influenciado a inovação nas empresas.

A partir desses estudos, as principais conclusões a que os pesquisadores chegaram é que a comunicação interna:

1. Auxilia na disseminação e no enriquecimento de ideias, fazendo que as ideias de inovação se espalhem entre os funcionários, que podem discuti-las e refiná-las, acrescentando ou retirando nuances que podem ajudar a melhor responder a um problema; 
2. Impacta a disseminação dos conhecimentos necessários para garantir a efetividade do processo de inovação: neste sentido, torna-se estratégica ao fazer as habilidades, técnicas e ferramentas necessárias para trabalhar as ideias inovadoras serem conhecidas pelos funcionários, tornando-os mais efetivos no processo;

3. Engaja e motiva os funcionários tanto em seus trabalhos quanto durante o processo de inovação: durante a execução das ações para o desenvolvimento de inovações, a comunicação interna exerce papel fundamental ao manter os funcionários engajados para que possam finalizar o processo obtendo os melhores resultados possíveis;

4. Influencia as relações sociais e a troca de informações entre as pessoas envolvidas na inovação: neste aspecto, a comunicação interna é responsável por garantir que os indivíduos envolvidos no processo de inovação tenham as metodologias e as ferramentas necessárias para interagirem e se relacionarem, de modo que possam se alinhar e se manter no mesmo nível de conhecimento sobre o processo de inovação;

5. Deve ser específica e adaptada quando necessária no processo de inovação: para que a comunicação interna seja eficaz, é importante que ela seja planejada especificamente para o momento de inovação, escolhendo-se, assim, processos, métodos e ferramentas não só necessários, mas também adequados para facilitar o processo.

As publicações analisadas neste artigo, portanto, deixam claro que a comunicação interna pode impactar diversas fases do processo de inovação, como a difusão e fertilização de ideias, o alinhamento entre as etapas - ou módulos - do processo de inovação, o engajamento dos envolvidos e a aceitação dos resultados deste processo.

Assim, chega-se à conclusão de que a comunicação de fato impacta a inovação nas organizações. Sugere-se que as próximas contribuições para o tema trabalhem a construção de modelos, metodologias e frameworks de atuação da comunicação interna voltada à inovação, definindo processos e canais específicos.

\section{REFERÊNCIAS}

ARAÚJO, Carlos Alberto. Bibliometria: evolução histórica e questões atuais. Em Questão, Porto Alegre, v.12, n.1, p.11-32, 2006.

BOTELHO, Louise Lira R.; CUNHA, Cristiano Castro A.; MACEDO, Marcelo. 0 método da revisão integrativa nos estudos organizacionais. Gestão e Sociedade, Belo Horizonte, v.5, n.11, p.121-136, 2011.

BRUM, Analisa de Medeiros. Endomarketing de A a Z: como alinhar o pensamento das pessoas à estratégia da empresa. Rio de janeiro: Integrare, 2010.

BUCKLER, Sheldon A. The spiritual nature of innovation. Research-Technology Management, v.40, n.2, p.43-47, 1997. doi: https://doi.org/10.1080/08956308.1997.11671116.

CENERINI, Vanessa. As ferramentas da comunicação interna: um estudo sobre os veículos de comunicação nas organizações. 2009. Trabalho de Conclusão de Curso (Graduação em Comunicação Social) - Centro Universitário de Maringá, Maringá, 2009.

CLEMEN, Paulo. Como implantar uma área de comunicação interna: nós, as pessoas, fazemos a diferença. Rio de Janeiro: Mauad, 2005. 
CORRÊA, Elizabeth Saad. Comunicação digital e seus usos institucionais. In: KUNSCH, Margarida Maria (org.). Gestão estratégica em comunicação organizacional e relações públicas. São Caetano do Sul: Difusão, 2008. p.169-184.

CORAL, Eliza; GEISLER, Lisiane. Organização para a inovação. In: CORAL, Eliza; OGLIARI, André; ABREU, Aline França de. Gestão integrada da inovação: estratégia, organização e desenvolvimento de produtos. São Paulo: Atlas, 2008. p.45-82.

CROSSAN, Mary M.; APAYDIN, Marina. A multi-dimensional framework of organizational innovation: a systematic review of the literature. Journal of Management Studies, Durham, v.47, n.6, p.1154-1191, 2009.

DAMANPOUR, Fariborz. Organizational innovation: a meta-analysis of effects of determinants and moderators. Academy of Management Journal, New York, v.34, n.3, p.555-590, 1991.

DIGMAN, Lester A. Organizational factors related to technology transfer and innovation. The Journal of Technology Transfer, New York, v.2, n.1, p.61-68, 1977.

DRUCKER, Peter F. Prática da administração de empresa. 2ed. Rio de Janeiro: Fundo de Cultura, 1964.

EVANS, David; PEARSON, Alan. Systematic reviews: gatekeepers of nursing knowledge. Journal of Clinical Nursing, New Jersey, v.10, n.5, p.593-599, 2001.

FAIRTLOUGH, Gerard. Organizing for innovation: compartments, competences and networks. Long Range Planning, Amsterdam, v.27, n.3, p.88-97, 1994.

FRANÇOIS, Jean-Paul; FAVRE, Florent.; NEGASSI, Syoum. Competence and organization: two drivers of innovation. Economics of Innovation and New Technology, Abingdon-on-Thames, v.11, n.3, p.249-270, 2002.

FUCHIGAMI, Katsuyoshi. A study of organizational communication in a school during the pre- and post-innovation periods. The Japanese Journal of Psychology, New Jersey, v.64, n.1, p.35-42, 1993.

GARCÍA-MORALES, Víctor; MATÍAS-RECHE, Fernando; VERDÚ-JOVER, Antonio. Influence of internal communication on technological proactivity, organizational learning, and organizational innovation in the pharmaceutical sector. Journal of Communication, Oxford, v.61, n.1, p.150-177, 2011.

GLIŃSKA-NEWEŚ, Aldona et al. Innovations among people: how positive relationships at work can trigger innovation creation. Ekonomie a management, Liberec, v.20, n.3, p.84-100, 2017.

KUNSCH, Margarida. Planejamento de relações públicas na comunicação integrada. São Paulo: Summus, 2003.

LASSERSON, Toby J.; THOMAS, James; HIGGINS, Julian P. T. Starting a review. In: HIGGINS, Julia; THOMAS, James (ed.). Cochrane handbook for systematic reviews of interventions. Chichester: Cochrane, 2019.

LEMOS, Else. Comunicação interna como diferencial em relações públicas. In: FARIAS, Luiz Alberto de (org.). Relações públicas estratégicas: técnicas, conceitos e instrumentos. São Paulo: Summus, 2011. p.151-164. 
LINKE, Anne; ZERFASS, Ansgar. Internal communication and innovation culture: developing a change framework. Journal of Communication Management, Bingley, v.15, n.4, p.332-348, 2011.

MACARENCO, Isabel; TERCIOTTI, Sandra Helena. Comunicação empresarial na prática. 3.ed. São Paulo: Saraiva, 2013.

MYERS, Sumner; MARQUIS, Donald G. Successful industrial innovations. a study of factors underlying innovation in selected firms. Washington, DC: National Science Foundation, 1969.

ROTHWELL, Roy; DOGSON, Mark. External linkages and innovation in small and medium-sized enterprises. R\&D Management, Hoboken, v.21, n.2, p.125-138, 1991.

SUH, Jiwon; HARRINGTON, James; GOODMAN, Doug. Understanding the link between organizational communication and innovation: an examination of public, nonprofit, and for-profit organizations in South Korea. Public Personnel Management, Thousand Oaks, v.47, n.2, p.217-244, 2018.

TROTT, Paul. Gestão da inovação e desenvolvimento de novos produtos. 4.ed. Porto Alegre: Bookman, 2012.

ULIJN, Jan et al. Innovation, corporate strategy, and cultural context: what is the mission for international business communication? The Journal of Business Communication, Thousand Oaks, v.37, n.3, p.293-316, 2000.

ZHANG, Gang; GAO, Ruoyang. Modularity and incremental innovation: the roles of design rules and organizational communication. Computational and Mathematical Organization Theory, New York, v.16, p.171-200, 2010.

Artigo recebido em 19.10.2019 e aprovado em 22.10.2021. 